\title{
IoT Testbed Business Model
}

\author{
Edgar M. Silva',2, Pedro Maló1,2 \\ ${ }^{1}$ Faculdade de Ciências e Tecnologia (FCT), Universidade Nova de Lisboa (UNL), Caparica, Portugal. \\ ${ }^{2}$ UNINOVA, Centro de Tecnologia e Sistemas (CTS), Caparica, Portugal \\ Email: ems@uninova.pt, pmm@uninova.pt
}

Received 17 July 2014; revised 15 August 2014; accepted 15 September 2014

Copyright (C) 2014 by authors and Scientific Research Publishing Inc.

This work is licensed under the Creative Commons Attribution International License (CC BY).

http://creativecommons.org/licenses/by/4.0/

(c) (i) Open Access

\section{Abstract}

The Internet-of-Things (IoT) is today one of the hypes in the technological world but despite the enormous attention and research investment, the clear business value is still hard to perceive. IoT deployments are costly to be installed, managed and maintained, and need to provide a very clear value to justify the investments. For another viewpoint, IoT technologies need to be proven before deployment, which implies the need to test and assess IoT solutions in real settings and involve the actual target users. And as such, this presents an opportunity to have IoT deployments with a clear business model mainly focused on real-life large-scale research and technological experimentation. This would mean having a sustainable IoT infrastructure in-place based on the provision of experimentation services and a trial environment to industry and research, which then could also present an opportunity to establish added-value (business) services. This is the exact idea of the flagship SmartSantander testbed facility and especially its major deployment in the city of Santander, Spain. The SmartSantander facility business model is built around experimentally-driven research and technology development thus attracting many experimenters from industry and European research projects. This model makes it possible to sustain an outstanding large-scale IoT deployment of around 12,000 sensors and on top of it the development of new the development of new services and applications especially targeting the needs of users (citizens, businesses, authorities) in smart-cities. This paper studies the business model of outstanding SmartSantander facility in order to provide a generic Business Model for IoT testbeds that can provide guidance and be adapted by owners (or owners to-be) wishing to exploit their IoT deployments as facilities supporting experimentation and trials of IoT solutions.

\section{Keywords}

Internet-of-Things, Testbed, Business Model, SmartSantander

\section{Introduction}

The Internet-of-Things (IoT) is today a reality. Everyday flourishes new IoT deployments all over the world and 
in many areas, such as Smart Cities, Intelligent Transportation Systems, Domotics (Smart Buildings), and many others. The IoT is the idea of a global, dynamic network infrastructure where physical and virtual "things" (devices, sensors, smart objects, etc.) communicate and share information among each other in a highly heterogeneous environment. Several manufacturers (even key ones) are already engaged in developing new devices for many different purposes to target diverse application domains and services [1].

IoT is bringing diverse novel business models to the society due to its decentralized sensing and actuation capabilities, contributing also with high amount of data (big data), etc. Several cases could be mentioned, such as renting devices capabilities (e.g. testbeds) or the device itself (e.g. temperature sensor), selling information from either direct sensors data (e.g. orders tracking position) or already elaborated raw data (e.g. amount of free parking slots). These IoT technologies need however to be duly validated and assured and be hopefully with settings that closely relate to the target environments of such IoT services and applications.

In this sense several testbeds have been flourishing worldwide which can both support experimentation of new IoT technologies and solutions, support the research and development of new services and applications [2]. In Europe alone, several already exist for instance SmartSantander, Sunrise, Openlab, LOG-a-TEC, etc. that focus on IoT in disparate real-life settings and contexts of experimentation: smart-cities, smart-buildings, underwater, etc. Most of these have been co-funded under the scope of the FIRE (Future Internet Research Experimentation) initiative from the European Commission with the intention of establishing a working self-sustainable experimentation real-life living labs.

Such IoT testbeds need to be sustainable to be kept operational (even and especially after the funding has ended). As such, these have planned a clear exploitation path to the experimentation facility enclosing a realistic business model. Now, doing business (or at least paying out to the infrastructure operational costs) of providing experimentation and trials services is not a simple work as many variables and issues need to be considered. This work intends to provide some initial guidance and support to make business out of experimental IoT infrastructures and deployments.

In summary, this document intends to be a hitchhiker's guide, especially for IoT deployment owners and managers, by presenting them with a generic business model to make business out of an IoT deployment as an experimentation facility —an IoT Testbed. The model considers the four main aspects of a business: Infrastructure, Value proposition, Customers and Financials.

The generic business model is worked out based on the business ideas and commercialization principles behind the world-class SmartSantander Testbed and especially its major deployment in the city of Santander (Spain) as a clear and working business case of an IoT Testbed. In this way, readers are also provided with a view of the scheme and operation of real and active large-scale IoT Testbed deployment.

The following sections are divided as follows. Chapter 2 gives a general idea of what can be an IoT Testbed and what it has to offer. Chapter 3 presents the IoT Testbed Business Model development, explaining the different phases in the development process of the business model, and introduces the Business Model Canvas and its main areas used present the business model. Chapter 4 characterizes a practical and existing example, the SmartSantander IoT Testbed. Chapter 5 presents the IoT Testbed Generic Business Model which can be used by stakeholders as guidance to build their own IoT Testbeds, and finally Chapter 6 provides final considerations and possible future work trends based on the presented Business Model.

\section{Concept of an IoT Testbed}

IoT Testbeds serve as incubators for the Internet-of-Things (IoT), providing to companies and groups the necessary infrastructures in which ideas, technologies and best practices are tested and validated. In the past few years a necessity has emerged to experiment technologies, services, etc. in a more realistic environment, leading to the creation of new spaces rather than maintaining the research in the current IoT facilities, which are small, old laboratories and with a very controlled environment.

A Testbed is considered to be a structure (hardware, software or both) for experiments of wide-scale deployments. Constantly Humans perform their discoveries through experimentation, early and realistic testing is necessary in any kind of research, large-scale or not. IoT Testbeds have emerged focusing on different domains, as smart buildings, smart cities, environmental monitoring, security and surveillance, health-care, etc. relying on the utilization of innovative IoT technologies to improve the quality of the Humans life.

The Figure 1 displays an example of an IoT Testbed architecture which is divided in the IoT Environment 


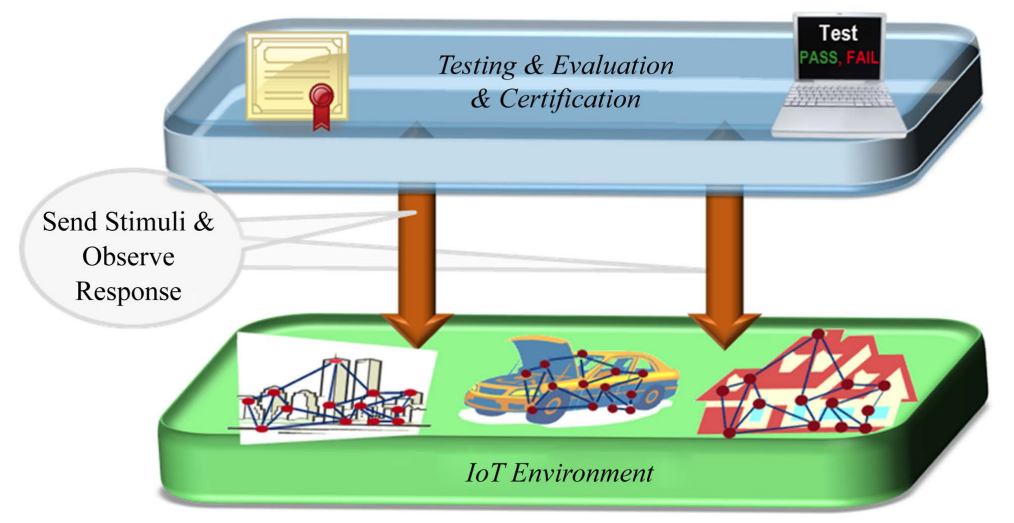

Figure 1. Concept of an IoT testbed.

layer that contain the network of IoT devices, repeaters and gateways, were the Authentication, Authorization and Accounting (AAA) systems are located, experimental support, management support (resource, configuration, etc.), application support, etc. The Testing \& Evaluation \& Certification layer is where the services are provided to the customers.

A Testbed facility should be able to provide true field experiments for architectures, key enabling technologies, services and applications, as well as IoT evaluation and certification in the context for what they were built for.

\section{IoT Testbed Business Model Development}

Business Model is a common word in the Management world, but in the last years it has been raising considerable interest for researchers [3]. The business model is seen as a good starting point for business creation or restructuring an existing one. Therefore, it can work as a helper to line up "technology development and economic value creation" [4].

Several definitions have been made along the years of the expression "business model", but a common definition is missing [5], next it's presented two of them which we believe that better exposes what is a business model. It can be defined as "An architecture for the product, service and information flows, including a description of the various business actors and their roles; as a description of the potential benefits for the various business actors; and a description of the sources of revenues" according to [6], another definition is: "A Business model describes the rationale of how an organization creates, delivers, and captures value" [7].

To describe a business model it is needed a common base of work, a type of language that everyone understands, which can be easily managed, facilitates descriptions and discussion. The Figure 2 displays the framework that will be used to present the IoT Testbed business model, this framework is based on the "business model canvas" work produced by [7]. This selection is grounded by the proof given in practice of the applicability of the model as for the innumerous references in publications (e.g., [8]).

\subsection{Business Model Development Process}

The development process of the business model was composed by two stages, an initial stage (during the first project year-FP7-288315 PROBE-IT "Pursuing ROadmaps and BEnchmarks for the Internet of Things") in which the authors identify key aspects regarding the deployments of IoT Testbeds through the analysis of existing roadmaps (e.g. IoT strategic research roadmap, vendor roadmaps market views, Artemis roadmap, IoT-I, etc.), through the attendance of workshops, conferences and even by strategic research agendas from Europe, South America, Africa and China. Having identified the essential trends, themes, a critical thinking phase was initiated in order to point out the main drivers.

The second and final stage occurred during the second project year in which all the business model aspects were tuned, narrowing out any existing doubts. This was achieved through several presentations in workshops, namely the IoT World meets in Brazil event (held in São Paulo, Brazil), IoT-Week in Helsinki and at the IoT Interoperability Workshop (held in Beijing, China). The workshops allowed the interaction with stakeholders, 


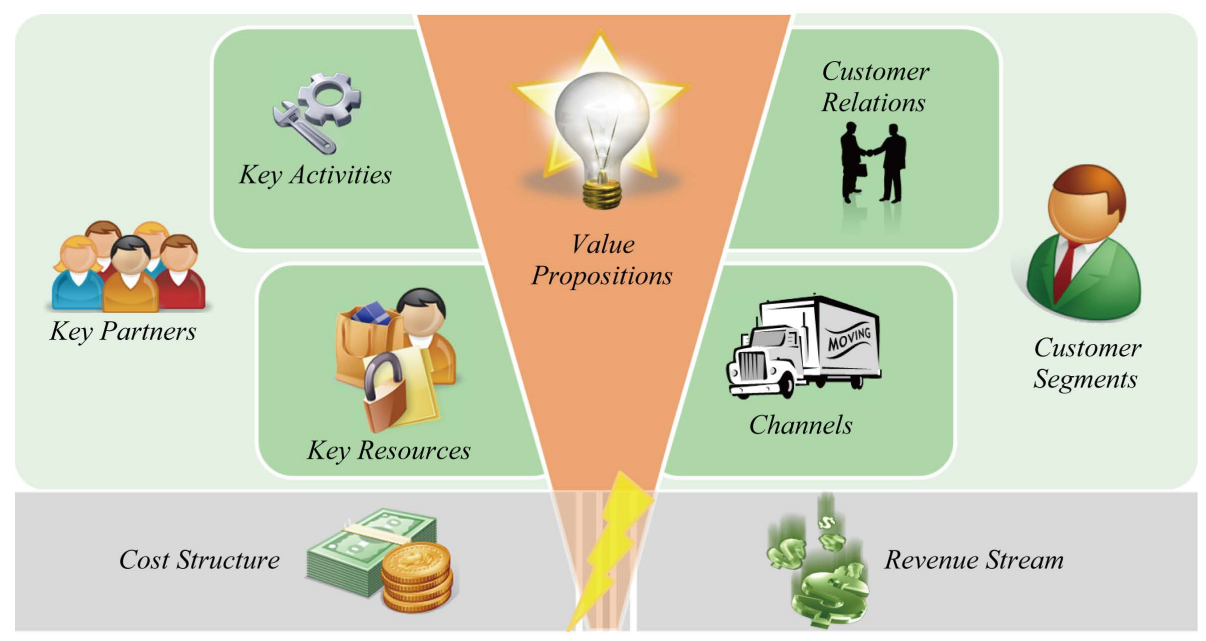

Figure 2. Business model canvas, as from [7].

experts and the scientific community. Likewise, IoT deployment pioneers were also consulted to retrieve ideas, opinions on Smart environments and their critical perspective on the document addressed topics.

The business model validation was performed during the development process throughout a constant interaction with different clusters of persons, namely the internal consortium (i.e. Probe-IT partners) through various discussions to analyze results and improve, review, restructure, and refine of the business model. Another validation cluster was the European Research Cluster on the Internet-of-Things (IERC) Activity Chains (AC), in which were carried out concertations activities with relevant European projects (e.g. IoT-A, IoT-I) towards the consolidation of the business model. And also the IoT community cluster that embraces activities such as interops, conferences, workshops, etc. in order to facilitate the interaction with a wider group of different experts, as has been mentioned above.

The development process also took advantage of several contributions. Luis Muñoz from SmartSantader which is the European flagship project in IoT for smart cities; Jian MA from Wuxi smart city which is the lighthouse project in Wuxi new district, China, in IoT for smart cities; Louis Coetzee connection to Johannesburg Broadband Network Project (JBNP) which is the urban networking project of reference in South Africa, Gabriel Marão a connection to IBM Smarter Cities in Brazil for the development of smart cities in Rio de Janeiro; and Sébastien Ziegler from HOBNET project/platform for smart buildings.

\subsection{Business Model Representation: Canvas}

The business model canvas (see Figure 2) is divided in four main areas of a business: Infrastructure, Value proposition, Customers and Financials, where the Infrastructure is subdivided in three themes: Key Partners, Key Activities and Key Resources. The Customer main area is also sub-divided in three themes: Customer Relations, Channels and Segments; on the other hand the main area of Financial is sub-divided in two themes: the Cost Structure and Revenue Stream.

In the following paragraphs each one of the identified themes will be explained in more detail, and is also important to emphasize the fact that each one of the themes is influenced by the others, i.e. they are not standalone. The Infrastructure area works as foundation for business, composed by Key Partners, Activities and Resources. Suppliers and/or partners getting together with a common goal, creating alliances to improve their business model, to reduce costs, reduce the risks and uncertainty, to get specific resources and activities or even to create a new business, forms the Key Partners theme.

Key Activities define the more important things a partner must do, in order to make the business model work. These activities can be of different types depending on what the business as to offer, it can be a problem solving if the objective is to offer consultancy, or a supply chain management if it is hardware production or in case of software provider the activity is the software development. Others could be identified depending of the value proposition, for example keeping the sustainability of a platform/network. Every business model needs some kind of resources to create value to customers, Key Resources are considered as essential assets to maintain and 
support the business. They can be of different type such as intellectual, financial, human or physical, as also they can be provided by key partners as owned or leased by the company.

Value Proposition area specifies a collection of products, services or benefits that the company can offer to meet some customer's needs. In this sense, the more the customer needs are fulfilled higher will be the chances of the company's value proposition being selected. Value Proposition can offer a new, innovative or disruptive product as well a similar product but with additional attributes and features, i.e. a Value Proposition creates value for Customers Segments.

The Customers area is formed by Customers Segments, Relations and Channels, is mainly the heart of a business model. No business can prevail without customers, therefore a company must select the clients or the group of clients wisely, i.e. which Customer Segments will serve. A business model can identify more than one Customer Segment meaning that each segment can focus in client groups with common behaviors, common needs or with other aspects.

Having identified the Value Proposition and the target market, is necessary to know how the product is delivered, publicize, in what way can clients be informed about the product, services and company existence, or even helping customers in the evaluation of the Value Proposition, summing it up, how the communication between clients and company can be performed. This topic is covered by the Channels theme which can be executed by the company as by partners. Customer Relationship characterizes the way a company interacts with clients, this interaction could be in a more familiarly way (e.g. individual assistance) or automated (e.g. selfservice) depending on the approach taken by the company.

Settled the stakeholders, the stakeholder's actions and the benefits it is time to make reference to the Financial area, where all of this stands on. As stated previously Financial area is sub-divided in two themes, Cost Structure and Revenue Stream. The Cost Structure defines the most important costs inherent to the business. These costs can be easily calculated after specifying who are the Key Partners, Key Activities and Key Resources. On the other hand the Revenue Stream represents the capability of generating money. This can be achieved through different types of revenue such as fees, advertising, sales, renting, etc.

\section{SmartSantander IoT Testbed Business Model}

By means of a practical and existing example was chosen the SmartSantander, which is an experimental test facility at a world city scale. SmartSantander provides a unique-in-the-world platform for IoT experimentation and evaluation under realistic operational conditions in a large-scale scenario (see Figure 3).

The SmartSantander facility supports experimentation and research in the Internet-of-Things (IoT) scope regarding architectures, enabling technologies, services and applications for smart cities [9]. Allowing users to test high-level services based on the data provided by the sensor network, to test sensor network algorithms, multihop protocols, radio interfaces, and key support services such as discovery, identity management and security. Another key aspect is the evaluation of social acceptance of IoT technologies and services.

The SmartSantander IoT Testbed sensor network deployment (of 12,000 sensors) is done in electrical poles,

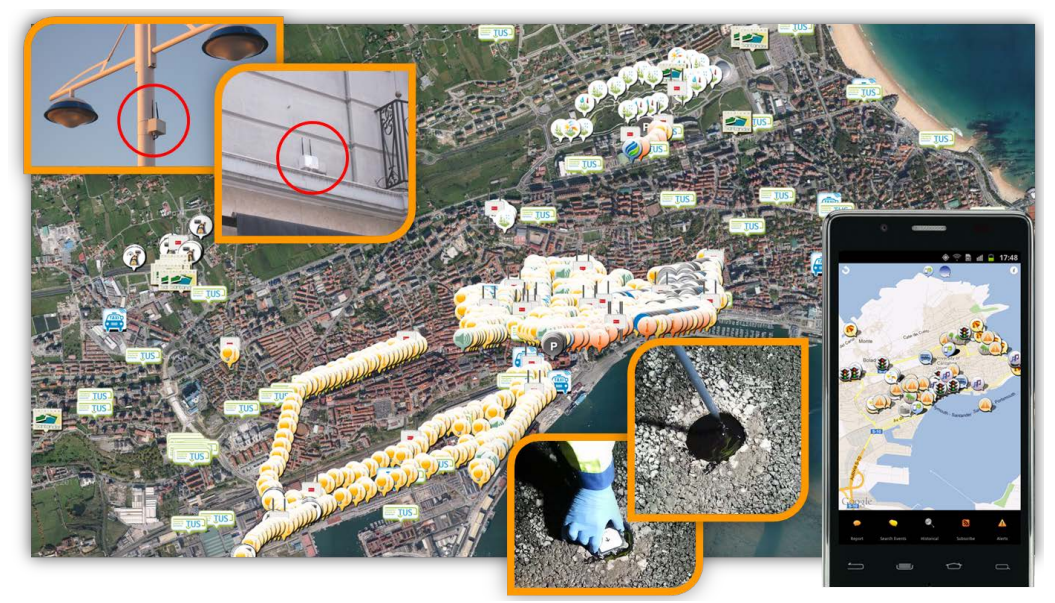

Figure 3. SmartSantander IoT testbed, Santander deployment. 
building walls, bus, taxis, parking lots (on the ground), garbage bins, etc. The deployed sensors are capable of traffic monitoring as free parking lots checking, acoustic-based monitoring and environment surveillance (detection of emergency cars, traffic density monitoring, etc.), as well as participatory sensing. Figure 3 shows a satellite picture of Santander City with the sensors location.

Towards the chosen IoT Testbed example, SmartSantander, and with the presented business model canvas, the authors will describe next the four main areas of the business model: Infrastructure, Value proposition, Customers and Financials (see Figure 4). The SmartSantander has the Santander Bank as strategic financial partner also the Santander Ayuntamiento (council) has a role in this aspect as in logistics. The Universidad de Cantabria is the strategic system integrator, concerning technology the TST (as strategic) and Libelium are the selected providers. The Certification aspect is being treated at the moment with ETSI (European Telecommunications Standards Institute) since it's the leading organization in standardization and certification, and therefore one of the desired partners. The Data Store \& Data Service is assured by Telefónica through its IDAS-Intelligence Data Advanced Solution platform, and as coordinator the SmartSantander organization.

Key Activities consist in the infrastructure operation performed by the coordinator and Telefónica, infrastructure maintenance actions are done by Uni. Cantabria, the development of Testbed software (middleware and the Portal) is the University Cantabria responsibility. The provision of user support and management of the Infrastructure is performed by the coordinator. The Key Resources are identified as the personnel and technicians for the SmartSantander maintenance, development, operations, marketing, management, and for the user support teams. The energy sources (throughelectric poles) are provided by Santander council, the financial support is assured by the Santander Bank. The Testbed hardware is provided by TST and Libelium partners.

According to the raised activities and resources the costs are divided in an initial phase, with the SmartSantander planning, assembly of the Testbed and with organization costs. Afterwards the costs are with testing and validation techniques, maintenance and user support, expansion/renovation of the Testbed and in the on-going organizational costs.

For Customer Relations, SmartSantander presents its brand, discussion forums and online support in order to interact with its customers that are identified as IoT application developers, IoT hardware and communication providers, IoT research communities, IoT related certification authorities, project partners and open calls partners. The communication Channels are the IoT related communities, International and National project clusters, Testbed aggregator and general dissemination (videos, internet, etc.). The revenue is funneled through renting the Testbed to experimentations, for certification of solutions, validation and certification of IoT solutions and funding's by International and National research projects.

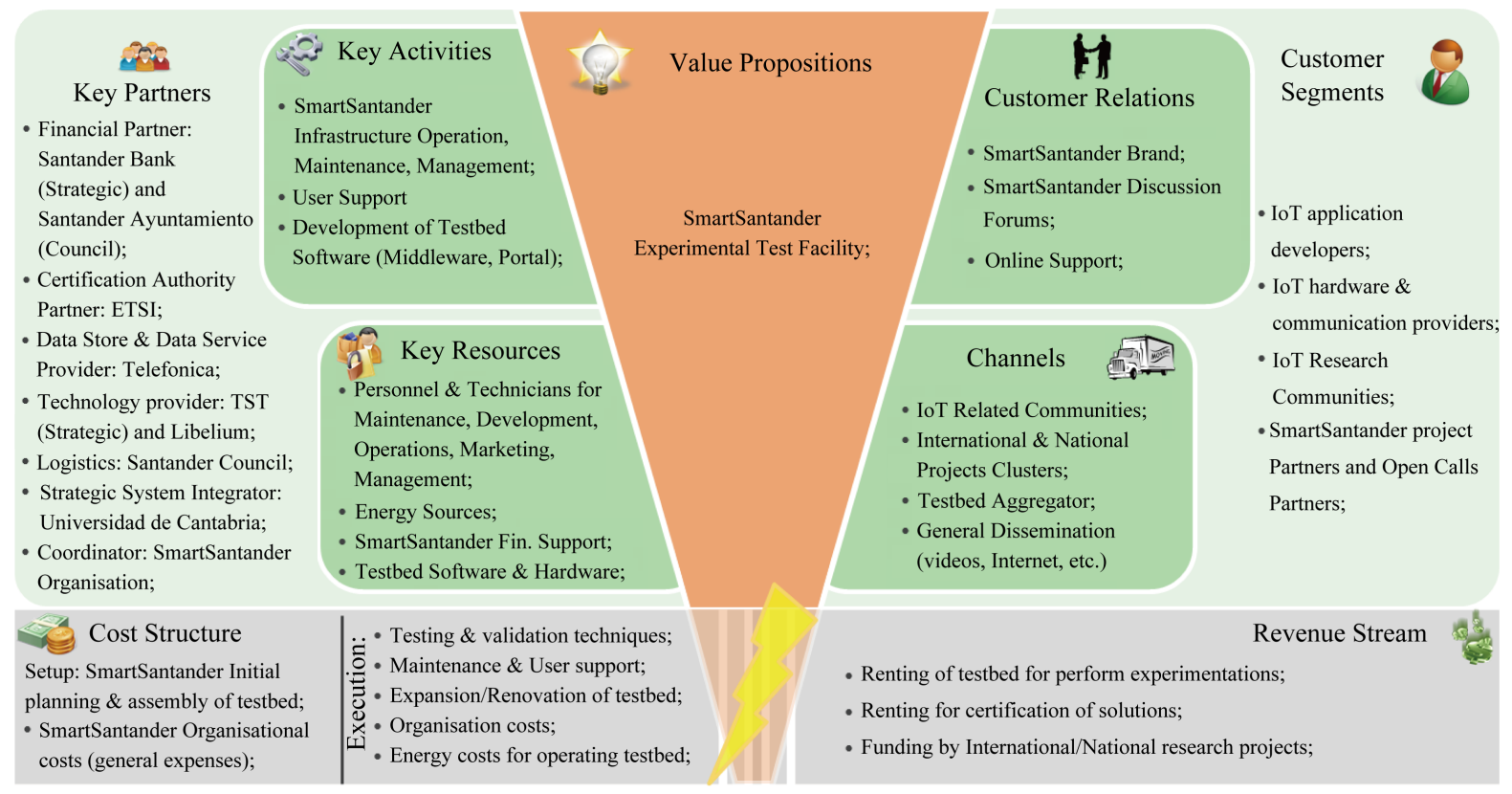

Figure 4. SmartSantander IoT testbed business model. 


\section{IoT Testbed Generic Business Model}

The existence of an IoT Testbed encourages stakeholders to perform tests, validation and certification of their IoT concepts, technologies and applications. Based on the introduced canvas (see Figure 2) the authors will present their interpretation of what should be the generic IoT Testbed business model. Figure 5 illustrates the generic IoT Testbed business model using the presented canvas which stakeholders can instantiate for a specific IoT Testbed case.

The existence of a generic IoT Testbed business model provides thoughtful guidelines for potential IoT Testbed owners, helping them in strategic decisions and plans in how they should perform their investment. Providing also consolidated views and perspectives regarding possible target markets and regarding all the costs involved.

A business model is composed by partners and their roles, which produce certain value to customers to achieve some financial gain, but of course it also has its costs. As key partners the authors identified several possible actors, namely a strategic and non-strategic financial partners for the monetary support since every business needs to have an investment at an early stage (and not only) and in this case the bigger the Testbed the bigger the investment needed. Strategic and non-strategic technology providers to supply the necessary equipment for the Testbed, logistics partners to manage material, equipment, staff, etc., experimentation data storage and data service provider so the generated data can be held and give to users as well as the right accesses. Strategic system integrator to get all the different parts in the system to work as a whole, coordination partner normally performed by the individual/company that got the big vision, certification authority partner and testing and validation technology partner as an certification and validation supplier. From these actors are expected specific activities and resources.

The activities are the infrastructure operation, maintenance and its management; development of the Testbed software; user support and validation and certification. The resources are personnel and technicians teams for maintenance, development, operations, marketing, management and user support; financial support; energy sources and the necessary Testbed software and hardware.

For the customer side were identified as probable clients the IoT application developers and IoT hardware and communication providers that gladly embraces this opportunity to test their own applications, hardware and communication protocols in a real life environment. Also the IoT research communities and IoT related certification authorities are also possible clients. To reach them, channels were identified, namely the IoT related communities, International and National project clusters, Testbed aggregator and general dissemination (videos,

\begin{tabular}{|c|c|c|c|c|c|}
\hline \multirow{2}{*}{$\begin{array}{l}\text { Key Partners } \\
\text { - (Strategic) Financial Partner; } \\
\text { - (Strategic) Technology } \\
\text { provider; } \\
\text { - Logistics Partner; } \\
\text { - Experimentation Data Store \& } \\
\text { Data Service Provider; } \\
\text { - Strategic Systems Integrator; } \\
\text { - Coordination Partner; } \\
\text { - Certification Authority Partner; } \\
\text { - Testing \& Validation } \\
\text { Technology Provider; }\end{array}$} & $\begin{array}{l}\text { Key Activities } \\
\text { - Operation of Infrastructure; } \\
\text { - Maintenance of Infrastruct; } \\
\text { - Development of Testbed } \\
\text { Software; } \\
\text { - User Support; } \\
\text { - Management of Infrastruct; } \\
\text { - Validation \& Certification; } \\
\end{array}$ & \multicolumn{2}{|c|}{$\begin{array}{c}\text { Value } \\
\text { Propositions }\end{array}$} & $\begin{array}{l}\text { Customer } \\
\text { Relations } \\
\text { - Branding; } \\
\text { - Discussion Forums; } \\
\text { - Online Support; } \\
\text { - Innovation office; }\end{array}$ & \multirow{2}{*}{$\begin{array}{l}\text { - IoT application developers; } \\
\text { - IoT hardware \& } \\
\text { communication providers; } \\
\text { - IoT Research Communities; } \\
\text { - IoT Related Certification } \\
\text { authorities; }\end{array}$} \\
\hline & $\begin{array}{l}\quad \text { Key Resources } \\
\text { - Personnel \& Technicians; } \\
\text { - Financial Support; } \\
\text { - Energy Sources; } \\
\text { - Testbed Software \& } \\
\text { Hardware; }\end{array}$ & \multicolumn{2}{|c|}{$\begin{array}{l}\text { Internet-of -Things } \\
\text { Infrastructure for } \\
\text { Testing \& } \\
\text { Evaluation \& } \\
\text { Certification for } \\
\text { Iot Cibcepts/ } \\
\text { Technologies/ } \\
\text { Applications }\end{array}$} & $\begin{array}{l}\text { Channels } \\
\text {-IoT Research Communities; } \\
\text { - International \& National } \\
\text { Projects Clusters; } \\
\text { - Testbed Aggregator; } \\
\text { - General Dissemination } \\
\text { (videos, Internet, etc.) }\end{array}$ & \\
\hline \multicolumn{3}{|c|}{ Cost Structure } & \multicolumn{3}{|c|}{ Revenue Streams } \\
\hline \multicolumn{3}{|c|}{\begin{tabular}{l|l} 
Setup: & Execution: \\
•Initial planning \& assembly of first & $\bullet$ Testing \& validat \\
testbed $\rightarrow$ Can be funded by public & $\bullet$ Maintenance of in \\
money if novel and if it's needed for & $\bullet$ User support for e \\
research; & • Energy costs for o \\
•Expansion of testbed; & • Expansion/Renov \\
• Organisation costs (general & • Organisation cost \\
expenses); & expenses);
\end{tabular}} & \multicolumn{3}{|c|}{$\begin{array}{l}\text { - Renting of test-bed for perform experimentations; } \\
\text { - Renting for certification of solutions; } \\
\text { - Validation and Certification of IoT solutions; } \\
\text { - Funding by Internation/National research projects; }\end{array}$} \\
\hline
\end{tabular}

Figure 5. IoT testbed generic business model. 
internet, etc.). Interaction (customer relations) is left to discussions forums, online support, brand creation and the innovation office.

As per costs inherent to the Testbed business model were recognized two stages, the infrastructure setup and the maintenance (execution). In the setup stage, costs can be related with the initial planning and assembly of the prime Testbed (which can be founded by public money if novel and if it's needed for research), the Testbed expansion, organization costs (general expenses); in the execution stage is identified costs with testing and validation techniques, infrastructure maintenance, user support for experiments, energy costs to operate the Testbed, expansion/renovation of Testbed and also the organization costs. Through the value proposition, Internet-ofThings Infrastructure for Testing \& Evaluation \& Certification for IoT Concepts/Technologies/Applications is expected to accomplish revenue by renting the Testbed to perform experimentations, renting for solutions certification, the provision of validation and certification of IoT solutions, and through funds by the International/ National research projects.

\section{Conclusions}

The Internet-of-Things is here to stay: IoT deployments abound, more IoT-related technologies appear and new IoTcentric apps are launched each day. However, the IoT movement still looks a bit unstructured which in some sense is hindering mass adoption: there is a lack of standards at many levels of the IoT (communication, data, services, middleware, semantics, etc.) and especially there is a lack of novel business models built around the strengths of the IoT. And of course, killer business model(s) for the IoT would be just perfect.

Looking things from another angle, it is needed to experiment the Internet-of-Things solutions all the way through the development process. And also to make sure that solutions work and are well-accepted by users, it is crucial to experiment and trial the IoT technologies in real-life large-scale environments that closely match that of application. And as it happens, the IoT is a market where many small-to-medium enterprises (even sole individuals) are developing their solutions and they do not have the economic capacity to setup testing infrastructures. They will need to rent some infrastructure for this.

IoT testbeds provide exactly this: an infrastructure where experimenters can deploy and execute their experiences and trials, collect data and further improve their IoT-based solutions (products, services, algorithms, protocols, etc.). In Europe, outstanding IoT infrastructures have been setup to provide real-life large-scale experimentally-driven research and development services. And of major importance, the SmartSantander testbed facility is the lighthouse smart-city IoT testbed in Europe with around 12,000 sensors deployed.

The SmartSantander IoT testbed core business model is based on renting experimentation services and then also on services that exploits the testbed deployments. A full-fledged experimentation/service platform is in place to manage the whole SmartSantander infrastructure and assure a non-stop operation, to schedule experiments and to move data to a place where experimenters can be retrieve, analyze and study.

Now, making sustainable business out of an IoT test-bed is not a trivial task. There is an operational structure to support it, and there is the issue about the energy costs, the maintenance and replacement of the IoT nodes, the software platform to evolve and correct in case of bugs, provision of support to experimenters including the experimentation interfaces, etc. There are also many key partners in the endeavor, also suppliers of hardware, networking, services, etc. Add up the costs to promote the facility and attract experimenters and engage users to the tests. Finally, there are the revenue schemes to be applied in order to make it a sustainable business.

Understanding this, the authors provided here a generic business model inspired in the very complete SmartSantander testbed facility business model. The generic business model considers the four main aspects of a business - Infrastructure, Value proposition, Customers and Financials—and thoroughly details all the elements of these in view of an IoT testbed business.

The generic business model for IoT testbeds especially targets IoT deployment owners (or owners to-be) and managers presenting them a starting point to inspect, analyze and adapt if interested in making business out of an IoT deployment in the form of an experimentation facility. The business model case study of the SmartSantander facility enables stakeholder to fully understand the elements in the generic business model and to see what considerations should be met while moving forward on creating a commercially sustainable IoT testbed.

\section{Acknowledgements}

We would like to thank all the partner of the FP7-288315 Probe-IT consortium for their contribution in the dis- 
cussion and concertation activities, and also a special thank is given to Luis Muñoz for its insights and clarification regarding the SmartSantander IoT Testbed, its operational scheme and business models.

This work was supported in part by the European Commission funds under the framework of FP7-288315 Probe-IT and FP7-318381 EAR-IT support actions.

\section{References}

[1] Maló, P., et al. (2013) Deliverable D3.1b Roadmaps for IoT Deployments, FP7-288315 PROBE-IT. Pursuing ROadmaps and BEnchmarks for the Internet of Things.

[2] Gluhak, A., Krco, S., Nati, M., Pfisterer, D., Mitton, N. and Razafindralambo, T. (2011) A Survey on Facilities for Experimental Internet of Things Research. IEEE Communications Magazine, 49, 58-67. http://dx.doi.org/10.1109/MCOM.2011.6069710

[3] Morris, M., Schindehutte, M., Richardson, J. and Allen, J. (2006) Is the Business Model a Useful Strategic Concept? Conceptual, Theoretical, and Empirical Insights. Journal of Small Business Strategy, 17, 27-50.

[4] Chesbrough, H. and Rosenbloom, R. (2002) The Role of the Business Model in Capturing Value from Innovation: Evidence from Xerox Corporation's Technology Spin-Off Companies. Industrial and Corporate Change, 11, 529-555. http://dx.doi.org/10.1093/icc/11.3.529

[5] Morris, M., Schindehutte, M. and Allen, J. (2005) The Entrepreneur's Business Model: Toward a Unified Perspective. Journal of Business Research, 58, 726-735. http://dx.doi.org/10.1016/j.jbusres.2003.11.001

[6] Timmers, P. (1998) Business Models for Electronic Markets. Electronic Markets, 8, 3-8. http://dx.doi.org/10.1080/10196789800000016

[7] Osterwalder, A. and Pigneur, Y. (2010) Business Model Generation. Self-Published, 281.

[8] Guinard, D., Trifa, V., Mattern, F. and Wilde, E. (2011) From the Internet of Things to the Web of Things: Resource-Oriented Architecture and Best Practices. Architecting the Internet of Things, 97-129. http://dx.doi.org/10.1007/978-3-642-19157-2_5

[9] Muñoz, L., et al. (2011) Deliverable D1.1 First Cycle Architecture Specification, FP7-257992 SmartSantander. 
Scientific Research Publishing (SCIRP) is one of the largest Open Access journal publishers. It is currently publishing more than 200 open access, online, peer-reviewed journals covering a wide range of academic disciplines. SCIRP serves the worldwide academic communities and contributes to the progress and application of science with its publication.

Other selected journals from SCIRP are listed as below. Submit your manuscript to us via either submit@scirp.org or Online Submission Portal.
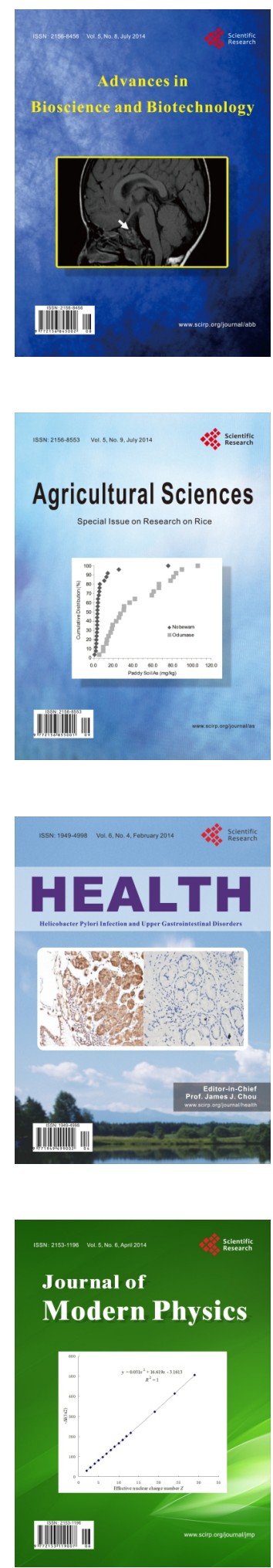
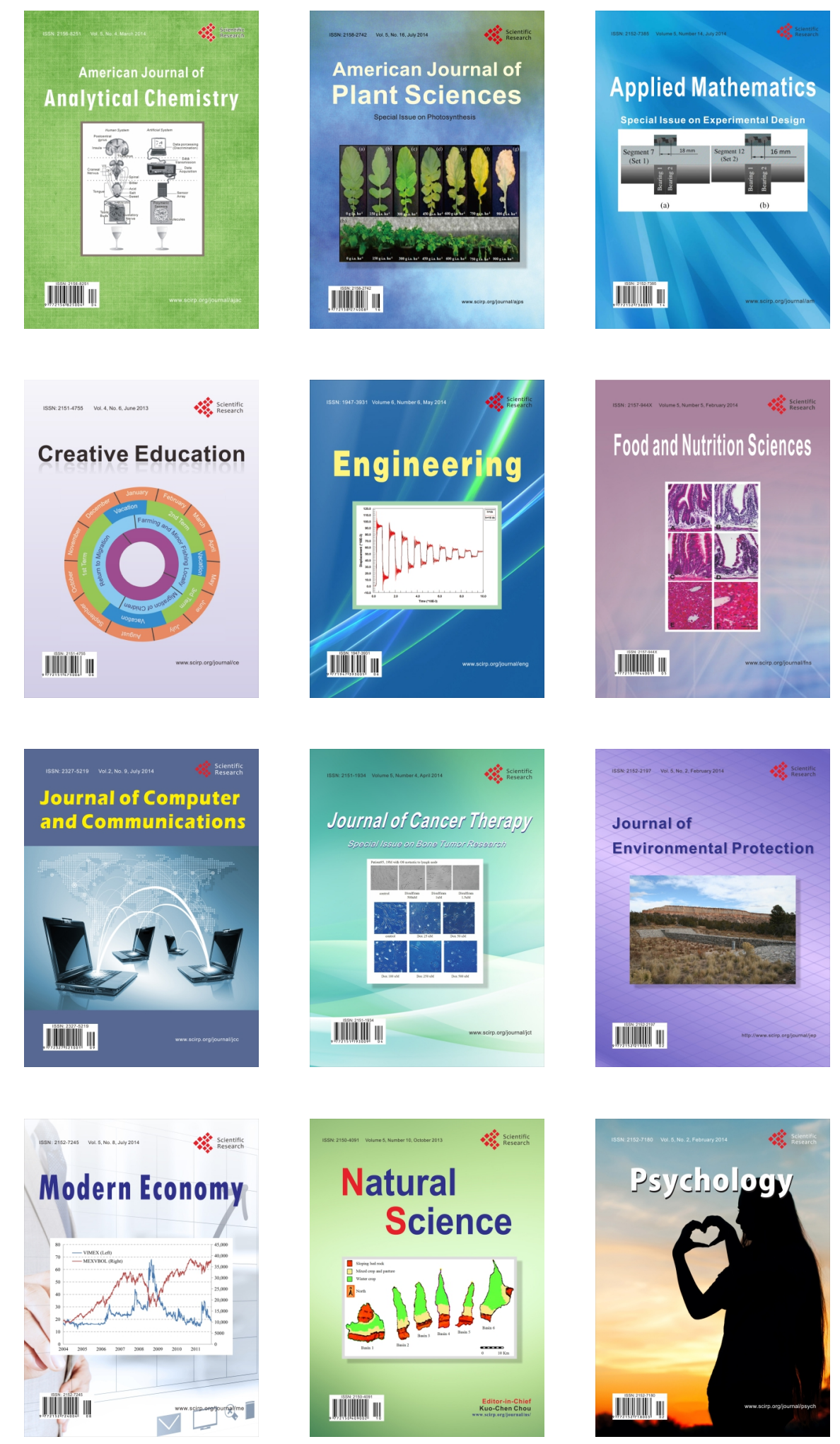